\title{
Anti-Tabak-Kampagnen und ihr Beitrag zur Gesundheitsförderung
}

\author{
K.-H. Jöckel ${ }^{1}$, K. Bromen ${ }^{1}$, B. Schmidt ${ }^{2}$, R. Rosenbrock ${ }^{3}$ \\ ${ }^{1}$ Universitätsklinikum Essen, Institut für medizinische Informatik, \\ Biometrie und Epidemiologie \\ ${ }^{2}$ Universität Bielefeld, Fakultät für Gesundheitswissenschaften \\ ${ }^{3}$ Wissenschaftszentrum Berlin, Arbeitsgruppe Public Health
}

\section{Comprehensive tobacco control programs can promote public health}

High smoking prevalences as well as the negative consequences for health are of concern and it is important to understand which tobacco control strategies are effective. This paper reviews different prevention strategies and its impact on peoples' perceptions, attitudes and behaviour regarding smoking. A large and comprehensive antitobacco control program, which combines the effects of personal, community-based and policy-orientated action, can influence smoking rates in a population.

\section{Zur Public Health-Relevanz des Rauchens}

Tabakkonsum ist der bedeutsamste mit dem Lebensstil assoziierte Risikofaktor für die Beeinträchtigung der Gesundheit.

Anti-Rauch-Programme verfolgen 3 Ziele:

- nichtrauchende Personen vom Rauchbeginn abzuhalten,

- bereits rauchende Personen vom Rauchen abzuhalten bzw. ihren Konsum einzuschränken,

- die Allgemeinbevölkerung vor unfreiwilliger Passivrauchexposition zu schützen.

\section{Verhaltensprävention}

Jugendtypischen Ansätzen kommt in der Verhaltensprävention zentrale Bedeutung zu.

\section{Gesundheitsalphabetisierung}

Wirksame jugendspezifische Prävention ist umfassend angelegt und enthält kognitive, affektive und behaviorale Elemente, da singuläre Maßnahmen Verhalten nicht verändern (Amer J Publ Hlth 1993; 83: 872-880). Maßnahmen der "sozialen Impfung" gelten als besonders wirksam (Hlth Educ Quart 1996; 23: 4864). Langfristige Verhaltensmodifikationen werden jedoch kaum erzielt (Hlth Educ J 1996; 55: 31-54). Zwar kann der Einstieg um 1-2 Jahre hinausgezögert, jedoch nicht völlig verhindert werden (J Adolesc 1999; 22, 583-599).

\section{Gemeindebezogene Maßnahmen}

Mehrdimensionale Programme erzielen Erfolge (Tobacco Control 2000; 9: 177186). Erfolgversprechend sind breit an- gelegte, evidenzbasierte, flexible und partizipative Maßnahmen, die begleitet werden von Veränderungen der politischen Rahmenbedingungen (Sowden/ Arblaster: Cochrane Review 2000).

\section{Massenmedienkampagnen}

Zusätzliche Unterstützung bieten Massenmedienkampagnen: Sie stimulieren die öffentliche Auseinandersetzung, verändern das gesellschaftliche Klima (Brit Med J 1996; 52: 108-120) und bereiten das Feld für weitere Maßnahmen (Hlth Educ J 1996; 55: 31-54); s. a. "Rauchfrei 2002«, S. 245. Gerade Anti-Tabak-Werbung ist erfolgversprechend (Tobacco Control 2000; 9: 47-63).

\section{Entwöhnungsmaßnahmen}

Aus ärztlicher Sicht kommen Maßnahmen zur Entwöhnung vorrangige Bedeutung zu. Da in Deutschland ca. 30\% der erwachsenen Bevölkerung rauchen und ca. $80 \%$ der Raucher gerne aufhören wollen (Zeitungsfund RR), sind fachgerechte Entwöhnungsprogramme notwendig (Sachverständigenrat SVR: Bedarfsgerechtigkeit Wirtschaftlichkeit 2001, III: 135-149). Hierzulande existieren verschiedene Entwöhnungsmaßnahmen (Kröger: Raucherentwöhnung in Deutschland 2000, BzgA Köln), allerdings sind sie selten hinreichend evaluiert.

Aus internationalen Studien ist bekannt, dass Selbsthilfemethoden im Unterschied zu Behandlungsmaßnahmen eine größere Reichweite besitzen und niedrigschwelliger sind, jedoch auch weniger effektiv (Treatment of Tobacco Use and Dependency 2000; Cochrane Database Syst 2000; CD001292). Erfolgreiche Behandlungsmaßnahmen basieren auf kognitiv-verhaltenstherapeutischen Elementen sowie einer ggf. ergänzenden $\mathrm{Ni}$ kotinsubstitution (Addict Behav 1996; 21: 699-707). Entwöhnungsprogramme
In Deutschland sterben jedes Jahr etwa 100000 Menschen an den Folgen ihres Konsums, vor allem an Krebs- und Herzkreislauferkrankungen (Sachverständigenrat SVR: Bedarfsgerechtigkeit Wirtschaftlichkeit 2001, III: 135-149). Die Mortalität summiert sich auf jährlich ca. 1,5 Mio. verlorene Lebensjahre; das sind 23\% aller vorzeitigen Sterbefälle von Männern und 6\% von Frauen. Allein durch Lungenkrebs in Folge des »Mitrauchens» sterben außerdem jährlich mindestens 400 Menschen.

Auch Kinder werden belastet: Erhöhte Auffälligkeiten finden sich u. a. bei Bronchitis, Pneumonie, Asthma, Mittelohrerkrankungen, plötzlichem Kindstod sowie Entwicklungsstörungen (SVR: Bedarfsgerechtigkeit Wirtschaftlichkeit 2001, III: 135-149). 
für Jugendliche sind noch nicht breit etabliert, und die bisherigen Erfolge sind bescheiden (J Amer Acad Child Adolesc Psychiat 2000; 39: 682-693).

Raucherentwöhnung kann gut im Rahmen der ärztlichen Versorgung durchgeführt werden. Kurze Ratschläge durch medizinisches Personal zeigen Effekte (Hlth Educ Quart 2000; 23: 4864; Cochrane Database Syst 2000; CD000165; Cancer Control 2000; 7: 5662). Etwas aufwändigere Maßnahmen, etwa eine motivierende Kurzberatung, sind noch wirksamer (Brit J Gen Pract 1999, 49: 611-616).

\section{(Nicht)Raucherschutz am Arbeitsplatz}

Entwöhnung und Rauchrestriktionen sind wichtige Instrumente der betrieblichen Gesundheitsförderung (Amer Econ Rev 1999; 89: 728-747). Nichtraucher weisen geringere Arbeitsfehlzeiten und höhere Produktivitätswerte auf (Tobacco Control 2001; 10: 233-238). Die Raucherprävalenzen und die täglich gerauchten Zigaretten bei den Beschäftigten sinken, wobei die Gefahr steigenden Konsums in der arbeitsfreien Zeit besteht. Außerdem steigen Aufhörmotivation (Prax klin Verhaltensmed Rehab 1997; 34: 122-133) und Entwöhnungsversuche. Langfristige Entwöhnungserfolge sind in rauchfreien Betrieben nicht unbedingt größer (Tobacco Control 2001; 10 : 267-272). Entwöhnungsunterstützung durch den Betriebsarzt sowie Nachsorge können die Rückfallprophylaxe verbessern (J Epidemiol Community Hlth 2000; 54: 349-354). Fehlende Compliance und kompensierbare Rauchbeschränkungen schwächen die Wirksamkeit (J Environ Occupat Med 1999; 41: 1121-1127).

\section{Verhältnisprävention}

Eine rasche Reduktion des Rauchverhaltens wird vor allem durch politische Entscheidungen erreicht. Ärztliches Engagement ist darum auch auf dem Feld der politischen Lobbyarbeit wünschenswert.

\section{Preis- und steuerbezogene Maßnahmen}

Preissteigerungen gelten als wirksamste Einzelmethode (Brit Med J 2000; 321: 358-361). Sie können effizient eingesetzt werden, um den Anteil der Konsumen- ten, die täglichen Konsummengen sowie den Einstieg in den Konsum zu verringern. Die Preiselastizität von Zigaretten wird in der Bevölkerung mit $-0,3$ und 0,5 angegeben, demnach geht eine Preiserhöhung von $10 \%$ mit Prävalenzreduktionen von 3-5\% einher (Nicotine \& Tobacco Research 1999; 1: 105-109).

\section{Tabakwerbung und -sponsoring}

Werbeverbote sind die zweite zentrale Maßnahme zur wirksamen Reduktion des Tabakkonsums (Brit Med J 2001; 322: 513-517). Sie können den Konsum verringern (u.a. Sucht 2001; 47: 104-113; J Adolesc 1999; 22: 583-599). Allerdings kann nur ein totales Werbeverbot wirksam Verhalten verändern, denn begrenzte Werbeverbote sind aufgrund kompensatorischer Möglichkeiten wirkungslos (J Hlth Econom 2000; 1117-1137).

\section{Inhalts- und Verpackungsgestaltung}

Produktregulative Maßnahmen sind darauf angelegt, die gesundheitsschädlichen Stoffe in Tabakerzeugnissen $\mathrm{zu}$ eliminieren und die Verpackung entsprechend zu gestalten. Ähnlich wie Beipackzettel von Arzneimitteln sollen Konsumenten $\mathrm{zu}$ informierten Entscheidungen befähigt werden. Auch wenn ihr verhaltensregulierendes Potenzial begrenzt ist, wird Produktregulation aufgrund von ethischen und Verbraucherschutz-Gründen befürwortet (Brit Med J 1996; 52: 108-120).

\section{Angebotsorientierte Maßnahmen}

Maßnahmen zur Angebotsreduktion gelten als weniger nützlich, die Ausnahme bilden die Anti-Schmuggelaktivitäten (Brit Med J 2000; 321: 358-361). Geschmuggelte Zigaretten erlauben aufgrund ihres günstigeren Preises gerade den besonders vulnerablen Gruppen den Konsum.

Die am weitesten verbreitete angebotsorientierte Methode ist das Verkaufsverbot an Jugendliche. Die Wirksamkeit solcher Verfügbarkeitsbeschränkungen ist gering, wenn immer wieder Lücken entstehen, die das Gesamtsystem destabilisieren (National Bureau of Economic Research 1996; 5740).

\section{Ausblick}

Die direkten und indirekten Kosten aufgrund tabakassoziierter Morbidität und Mortalität belaufen sich konservativ geschätzt auf jährlich 29-44 Mrd. Euro (Eur J Publ Hlth 2000; 40: 31-44), die Kosten einer Anti-Tabak-Kampagne in Höhe von ca. 25,6 Mio. Euro pro Jahr machen sich demnach selbst bei begrenzter Wirksamkeit bezahlt.

Eine umfassende Tabakprävention sollte auf drei Ebenen angesiedelt sein: Neben bevölkerungsweiten Strategien sollten zielgruppen- und settingspezifische Kampagnen sowie Maßnahmen der personalen Kommunikation, Beratung und Behandlung implementiert werden. Eine Kampagne wird dabei umso erfolgreicher, je mehr sie den Kriterien der Konsistenz und der Integration genügt (vgl. Z Gesundheitswissensch 1994; 3: 233-264). Ein Policy-Mix, in dem Angebote mit geringem staatlichen Eingriffscharakter und solche mit der Vermittlung eines positiven Lebensgefühls Vorrang vor solchen mit stärkerem Eingriffscharakter haben, verspricht den größtmöglichen Erfolg (Sachverständigenrat Bedarfsgerechtigkeit Wirtschaftlichkeit 2001, III: 135-149).

Für die ärztliche Praxis bedeutet dies, dass neben den Aktivitäten zur individuellen Unterstützung der Patienten die politische Unterstützungsarbeit für eine umfassende Tabakprävention einen wertvollen Beitrag zur Reduktion von vermeidbarer Krankheit und Tod leisten könnte.

Korrespondenz Dr. PH Bettina Schmidt Universität Bielefeld

Fakultät für Gesundheitswissenschaften Postfach 100131 33501 Bielefeld bettina.schmidt@uni-bielefeld.de

In der nächsten Folge lesen Sie: Krebsprävention mit Ernährung

\section{Fachliche Betreuung der} "Serie ... Präventionı:

Prof. Dr. Dr. h. c. Peter C. Scriba Medizinische Klinik / Klinikum Innenstadt der Universität München

Prof. Dr. Friedrich W. Schwartz Medizinische Hochschule Hannover Abteilung für Epidemiologie, Sozialmedizin und Gesundheitsforschung 